\title{
Large time behavior of solutions for the porous medium equation with a nonlinear gradient source
}

\author{
Nan Li ${ }^{1}$, Pan Zheng ${ }^{2,3^{*}}$, Chunlai $\mathrm{Mu}^{2}$ and Iftikhar Ahmed ${ }^{2}$
}

\section{"Correspondence:}

zhengpan52@sina.com

${ }^{2}$ College of Mathematics and

Statistics, Chongqing University,

Chongqing, 401331, P.R. China

${ }^{3}$ College of Mathematics and

Physics, Chongqing University of

Posts and Telecommunications,

Chongqing, 400065, P.R. China

Full list of author information is

available at the end of the article

\begin{abstract}
This paper deals with the large time behavior of non-negative solutions for the porous medium equation with a nonlinear gradient source $u_{t}=\Delta u^{m}+\left|\nabla u^{\prime}\right|^{q}$, $(x, t) \in \Omega \times(0, \infty)$, where $I \geq m>1$ and $1 \leq q<2$. When $l q=m$, we prove that the global solution converges to the separate variable solution $t^{-\frac{1}{m-1}} f(x)$. While $m<1 q \leq m+1$, we note that the global solution converges to the separate variable solution $t^{-\frac{1}{m-1}} f_{0}(x)$. Moreover, when $1 q>m+1$, we show that the global solution also converges to the separate variable solution $t^{-\frac{1}{m-1}} f_{0}(x)$ for the small initial data $u_{0}(x)$, and we find that the solution $u(x, t)$ blows up in finite time for the large initial data $u_{0}(x)$.
\end{abstract}

MSC: $35 \mathrm{~K} 55 ; 35 \mathrm{~K} 65 ; 35 \mathrm{~B} 40$

Keywords: large time behavior; separate variable solution; porous medium equation; gradient source; blow-up

\section{Introduction}

In this paper, we investigate the large time behavior of non-negative solutions for the following initial-boundary value problem:

$$
\begin{cases}u_{t}=\Delta u^{m}+\left|\nabla u^{l}\right|^{q}, & (x, t) \in \Omega \times(0, \infty), \\ u(x, t)=0, & (x, t) \in \partial \Omega \times(0, \infty), \\ u(x, 0)=u_{0}(x), & x \in \Omega,\end{cases}
$$

where $l \geq m>1,1 \leq q<2, \Omega$ is a bounded domain of $\mathbb{R}^{N}(N \geq 2)$ with smooth boundary $\partial \Omega$, and the initial function is

$$
u_{0}(x) \in C_{0}(\bar{\Omega})=\{z \in C(\bar{\Omega}): z=0 \text { on } \partial \Omega\}, \quad u_{0}(x) \geq 0, u_{0}(x) \not \equiv 0 .
$$

Equation (1.1) arises in the study of the growth of surfaces and it has been considered as a mathematical model for a variety of physical problems (see [1, 2]). For instance, in ballistic deposition processes, the evolution of the profile of a growing interface is described by the diffusive Hamilton-Jacobi type equation (1.1) with $m=l=1$ (see [3]).

One of the particular feature of problem (1.1) is that the equation is a slow diffusion equation with nonlinear source term depending on the gradient of a power of the solution. 
In general, there is no classical solution. Therefore, it turns out that a suitable framework for the well-posedness of the initial-boundary value problem (1.1) is the theory of viscosity solutions (see [4-6]), so we first define the notion of solutions.

Definition 1.1 A non-negative function $u(x, t) \in C(\bar{\Omega} \times(0, \infty))$ is called a solution of (1.1), if $u(x, t)$ is a viscosity solution to $(1.1)$ in $\Omega \times(0, \infty)$ and satisfies

$$
u(x, t)=0, \quad(x, t) \in \partial \Omega \times(0, \infty) \quad \text { and } \quad u(x, 0)=u_{0}(x) \in C_{0}(\bar{\Omega}), \quad x \in \bar{\Omega} .
$$

Under some assumptions, the global (local) existence in time, uniqueness and regularity of solutions to reaction-diffusion equations with gradient terms have been extensively investigated by many authors (see [7-12] and the references therein). In particular, in [1], Andreucci proved the existence of solutions for the following degenerate parabolic equation with initial data measures:

$$
\begin{cases}u_{t}=\Delta u^{m}+\left|\nabla u^{l}\right|^{q}, & (x, t) \in \mathbb{R}^{N} \times(0, \infty), \\ u(x, 0)=\mu, & x \in \mathbb{R}^{N},\end{cases}
$$

where $m \geq 1, q l \geq m, 0<q<2$, and $N \geq 1$.

The main purpose of this paper is to further study the large time behavior of nonnegative solutions $u(x, t)$ to (1.4) with homogeneous Dirichlet boundary conditions. In recent years, many authors have investigated the asymptotic behavior of solutions to the viscous Hamilton-Jacobi equations (see [3, 4, 7, 8, 10,13-21] and the references therein). For example, for the special case $m=l=1$, Gilding [22] studied the large time behavior of solutions to the following Cauchy problem:

$$
\begin{cases}u_{t}=\Delta u+|\nabla u|^{q}, & (x, t) \in \mathbb{R}^{N} \times(0, \infty) \\ u(x, 0)=u_{0}(x), & x \in \mathbb{R}^{N}\end{cases}
$$

and he gave the temporal decay estimates.

In [23], Stinner investigated the asymptotic behavior of solutions for the following one space dimensional viscous Hamilton-Jacobi equation:

$$
\begin{cases}u_{t}=\left(\left|u_{x}\right|^{p-2} u_{x}\right)_{x}+\left|u_{x}\right|^{q}, & (x, t) \in(-R, R) \times(0, \infty), \\ u( \pm R, t)=0, & t \in(0, \infty), \\ u(x, 0)=u_{0}(x), & x \in(-R, R),\end{cases}
$$

where $R>0, p>2$, and $1<q<p-1$, and he proved that these solutions converge to the steady states by Lyapunov functional.

In higher dimensional case, Barles et al. [24] studied the large time behavior of solutions for the following initial-boundary value problem:

$$
\begin{cases}u_{t}=\operatorname{div}\left(|\nabla u|^{p-2} \nabla u\right)+|\nabla u|^{q}, & (x, t) \in B(0,1) \times(0, \infty), \\ u(x, t)=0, & (x, t) \in \partial B(0,1) \times(0, \infty), \\ u(x, 0)=u_{0}(x), & x \in B(0,1),\end{cases}
$$

where $p \geq 2,0<q<p-1$, and they showed that the non-negative radially symmetric solutions converge to the stationary solution. 
Recently, in [25], Laurencot et al. extended the case $0<q<p-1$ of the problem (1.7) to the case $q \geq p-1$, and derived that these solutions converge to two different separate variables solutions according to the cases $q=p-1$ and $q>p-1$ in the general bounded domain $\Omega \subset \mathbb{R}^{N}$, respectively.

Motivated by the above work, by using the modified comparison argument, the selfsimilar transformation method, and the half-relaxed limits technique used in $[6,25,26]$, we investigate the asymptotic behavior of non-negative solutions to (1.1). Our main results in this paper are stated as follows.

Theorem 1.1 Let $l \geq m>1,1 \leq q<2$, and $l q=m$. Assume that $u_{0}(x) \in W^{1, \infty}(\Omega)$ satisfies (1.2). Then there exists a unique solution $u(x, t)$ to (1.1) in the sense of Definition 1.1 such that

$$
\lim _{t \rightarrow \infty}\left\|t^{\frac{1}{m-1}} u(t)-f\right\|_{\infty}=0
$$

where $f(x) \in C_{0}(\bar{\Omega})$ is the unique positive solution to

$$
-\Delta f^{m}-\left|\nabla f^{l}\right|^{q}-\frac{f}{m-1}=0 \quad \text { in } \Omega, \quad f>0 \quad \text { in } \Omega, \quad f=0 \quad \text { on } \partial \Omega .
$$

Moreover, we have $|\nabla u(t)| \in L^{\infty}(\bar{\Omega})$ for all $t \geq 0$ and

$$
\ell\left[u_{0}\right]:=\sup _{t \geq 0}\left\{\|\nabla u(t)\|_{\infty}\right\}<\infty .
$$

Theorem 1.2 Let $l \geq m>1,1 \leq q<2$, and $m<l q \leq m+1$. Assume that $u_{0}(x) \in W^{1, \infty}(\Omega)$ satisfies (1.2). Then there exists a unique solution $u(x, t)$ to (1.1) in the sense of Definition 1.1 such that

$$
\lim _{t \rightarrow \infty}\left\|t^{\frac{1}{m-1}} u(t)-f_{0}\right\|_{\infty}=0
$$

where $f_{0}(x) \in C_{0}(\bar{\Omega})$ is the unique positive solution to

$$
-\Delta f_{0}^{m}-\frac{f_{0}}{m-1}=0 \quad \text { in } \Omega, \quad f_{0}>0 \quad \text { in } \Omega, \quad f_{0}=0 \quad \text { on } \partial \Omega .
$$

Theorem 1.3 Let $l \geq m>1,1 \leq q<2$, and $l q>m+1$. Assume that $u_{0}(x) \in W^{1, \infty}(\Omega)$ satisfies (1.2) and suppose further that there exists $G_{0} \in W^{1, \infty}(\Omega)$ satisfying (1.2) such that

$$
u_{0}(x) \leq \frac{G_{0}(x)}{\ell\left[G_{0}\right]}, \quad x \in \bar{\Omega}
$$

where $\ell\left[G_{0}\right]$ is defined in (1.10). Then there exists a unique solution $u(x, t)$ to $(1.1)$ in the sense of Definition 1.1 such that

$$
\lim _{t \rightarrow \infty}\left\|t^{\frac{1}{m-1}} u(t)-f_{0}\right\|_{\infty}=0
$$

where $f_{0}(x) \in C_{0}(\bar{\Omega})$ is defined in (1.12). 
Theorem 1.4 Let $l \geq m>1,1 \leq q<2$, and $l q>m+1$. Assume that there exists a positive constant $K_{0}$ depending only on $m, l, q$, s, and $\varepsilon$ such that $\int_{\Omega} u_{0}^{s}(x) \phi_{\varepsilon}(x) d x \geq K_{0}$, where $s \in\left(0, \frac{1}{2}\right), \varepsilon \in(0,1)$, and $\phi_{\varepsilon}(x)=A_{\varepsilon} e^{-\varepsilon|x|}$ with $A_{\varepsilon}=\frac{1}{\int_{\Omega} e^{-\varepsilon|x|} d x}$. Then the solution $u(x, t)$ of the problem (1.1) blows up in finite time in the sense of weak solution. Moreover, the upper bound of blow-up time is given as follows:

$$
T \leq \frac{2}{q l-1}\left(\frac{q l}{q l+s-1}\right)^{\frac{q(m-1)}{q l-m}}\left(m \varepsilon^{2}\right)^{\frac{1-q l}{q l-m}} .
$$

Remark 1.1 Compared to the results in [25], we extend the results of $p$-Laplacian equation to the porous medium equation (1.1) with a nonlinear gradient source.

Remark 1.2 In Theorem 1.4, we only give an upper estimate of the blow-up time. But the lower estimate of the blow-up time is an open problem.

This paper is organized as follows. In Section 2, we establish the comparison lemmas to prove the uniqueness of the positive solution to (1.9) and the identification of the halfrelaxed limits. In Section 3, using the comparison principle, we construct the global solutions to obtain the upper bound and Hölder estimate of solutions to (1.1), and we prove Theorems 1.1 and 1.2 by the half-relaxed limits method. Moreover, we give the large time behavior of solutions to (1.1) with the small initial data $u_{0}(x)$ for $l q>m+1$, and we prove Theorem 1.3 in Section 4. Finally, we obtain the blow-up case, and we prove Theorem 1.4 in Section 5.

\section{Comparison lemmas}

In this section, we establish the following comparison lemma between positive supersolutions and non-negative subsolutions to the elliptic equation in (1.9): which is an important tool for the uniqueness of the positive solution to (1.9) and the identification of the halfrelaxed limits later.

Lemma 2.1 Let $l \geq m>1,1 \leq q<2$, and $l q=m$. Assume that $w \in U S C(\bar{\Omega})$ and $W \in$ $L S C(\bar{\Omega})$ are respectively a bounded upper semicontinuous (usc) viscosity subsolution and a bounded lower semicontinuous (lsc) viscosity supersolution to

$$
-\Delta \zeta^{m}-\left|\nabla \zeta^{l}\right|^{q}-\frac{\zeta}{m-1}=0 \quad \text { in } \Omega,
$$

such that

$$
w(x)=W(x)=0 \quad \text { for } x \in \partial \Omega,
$$

and

$$
W(x)>0 \quad \text { for } x \in \Omega
$$

Then

$$
w(x) \leq W(x) \quad \text { for } x \in \bar{\Omega} .
$$


Proof The proof is based on the idea as in [25,27], but with different auxiliary functions.

For $i \geq N_{0}$ large enough, it is easy to see that $\Omega_{i}=\left\{x \in \Omega: d(x, \partial \Omega)>\frac{1}{i}\right\}$ is a non-empty open subset of $\mathbb{R}^{N}$.

Since $\bar{\Omega}_{i}$ is compact and $W$ is lower semicontinuous, the function $W$ has a minimum in $\bar{\Omega}_{i}$. By the positivity (2.3) of $W$ in $\bar{\Omega}_{i}$, we have

$$
\mu_{i}=\min _{x \in \bar{\Omega}_{i}}\{W(x)\}>0 .
$$

Similarly, the compactness of $\bar{\Omega} \backslash \Omega_{i}$, the upper semicontinuity and boundedness of $w$ imply that $w$ has at least one point of maximum $x_{i}$ in $\bar{\Omega} \backslash \Omega_{i}$ and we set

$$
\eta_{i}=w\left(x_{i}\right)=\max _{x \in \bar{\Omega} \backslash \Omega_{i}}\{w(x)\} .
$$

It follows from $\partial \Omega \subset \bar{\Omega} \backslash \Omega_{i}$ and $w$ vanishes on $\partial \Omega$ by (2.2) that $\eta_{i} \geq 0$.

Next, we claim that

$$
\lim _{i \rightarrow \infty} \eta_{i}=0
$$

Indeed, owing to the compactness of $\bar{\Omega}$ and the definition of $\Omega_{i}$, there exist $y \in \partial \Omega$ and a subsequence of $\left\{x_{i}\right\}_{i \geq N_{0}}$ (not relabeled) such that $x_{i} \rightarrow y$ as $i \rightarrow \infty$. Since $w(y)=0$, we deduce from the upper semicontinuity of $w$ that

$$
\lim _{\varepsilon \rightarrow 0} \sup \{w(x): x \in B(y, \varepsilon) \cap \bar{\Omega}\} \leq 0 .
$$

Given $\varepsilon>0$ small enough, there exists $i_{\varepsilon}$ large enough such that

$$
x_{i} \in B(y, \varepsilon) \cap \bar{\Omega} \quad \text { for } i \geq i_{\varepsilon} .
$$

Therefore, we have

$$
0 \leq \eta_{i}=w\left(x_{i}\right) \leq \sup \{w(x): x \in B(y, \varepsilon) \cap \bar{\Omega}\}, \quad i \geq i_{\varepsilon} .
$$

Thus

$$
0 \leq \lim _{i \rightarrow \infty} \sup \eta_{i} \leq \sup \{w(x): x \in B(y, \varepsilon) \cap \bar{\Omega}\} .
$$

Letting $\varepsilon \rightarrow 0$, we conclude that zero is a cluster point of $\left\{\eta_{i}\right\}_{i \geq N_{0}}$ as $i \rightarrow \infty$. The claim (2.7) follows from the monotonicity of $\left\{\eta_{i}\right\}_{i \geq N_{0}}$.

Now, fix $s \in(0, \infty)$. For $\delta>0$ and $i \geq N_{0}$, we define

$$
z_{i}(t, x)=(t+s)^{-\frac{1}{m-1}} w(x)-s^{-\frac{1}{m-1}} \eta_{i}, \quad(t, x) \in[0, \infty) \times \bar{\Omega}
$$

and

$$
Z_{\delta}(t, x)=(t+\delta)^{-\frac{1}{m-1}} W(x), \quad(t, x) \in[0, \infty) \times \bar{\Omega} .
$$


It follows from the assumptions on $w$ and $W$ that $z_{i}$ and $Z_{\delta}$ are, respectively, a bounded usc viscosity subsolution and a bounded lsc viscosity supersolution to

$$
\partial_{t} \zeta-\Delta \zeta^{m}-\left|\nabla \zeta^{l}\right|^{q}=0 \quad \text { in }(0, \infty) \times \Omega
$$

and satisfy

$$
Z_{\delta}(t, x)=0 \geq-s^{-\frac{1}{m-1}} \eta_{i}=z_{i}(t, x), \quad(t, x) \in(0, \infty) \times \partial \Omega .
$$

Moreover, if

$$
0<\delta<\left[\frac{\mu_{i}}{1+\|w\|_{\infty}}\right]^{m-1} s
$$

then it follows from (2.5) and (2.8) that, for $x \in \Omega_{i}$,

$$
Z_{\delta}(0, x)=\delta^{-\frac{1}{m-1}} W(x) \geq \delta^{-\frac{1}{m-1}} \mu_{i} \geq s^{-\frac{1}{m-1}}\|w\|_{\infty} \geq z_{i}(0, x) .
$$

For $x \in \bar{\Omega} \backslash \Omega_{i}$, we deduce from (2.6) that

$$
Z_{\delta}(0, x) \geq 0 \geq s^{-\frac{1}{m-1}}\left(w(x)-\eta_{i}\right)=z_{i}(0, x) .
$$

By the comparison principle [6], we have

$$
z_{i}(t, x) \leq Z_{\delta}(t, x), \quad(t, x) \in[0, \infty) \times \bar{\Omega},
$$

for $i \geq N_{0}$ and $\delta>0$ satisfying (2.8).

According to (2.8), the parameter $\delta$ can be taken to be arbitrarily small in (2.9). Therefore, we deduce that

$$
(t+s)^{-\frac{1}{m-1}} w(x)-s^{-\frac{1}{m-1}} \eta_{i} \leq t^{-\frac{1}{m-1}} W(x), \quad(t, x) \in(0, \infty) \times \bar{\Omega},
$$

for $i \geq N_{0}$.

Passing to the limit as $i \rightarrow \infty$, it follows from (2.7) that

$$
(t+s)^{-\frac{1}{m-1}} w(x) \leq t^{-\frac{1}{m-1}} W(x), \quad(t, x) \in(0, \infty) \times \bar{\Omega} .
$$

Finally, let $s \rightarrow 0$ and take $t=1$; then we obtain

$$
w(x) \leq W(x), \quad(t, x) \in(0, \infty) \times \bar{\Omega} .
$$

The proof of Lemma 2.1 is complete.

A straightforward consequence of Lemma 2.1 is the uniqueness of the solution to (1.9).

Corollary 2.1 There is at most one positive viscosity solution to (1.9). 
By the similar argument, we have the following result to (1.12).

Lemma 2.2 Let $w \in U S C(\bar{\Omega})$ and $W \in L S C(\bar{\Omega})$ be respectively a bounded upper semicontinuous (usc) viscosity subsolution and a bounded lower semicontinuous (lsc) viscosity supersolution to

$$
-\Delta \zeta^{m}-\frac{\zeta}{m-1}=0 \quad \text { in } \Omega,
$$

satisfying (2.2) and (2.3). Then

$$
w(x) \leq W(x) \quad \text { for } x \in \bar{\Omega} .
$$

Proof The proof is similar as in Lemma 2.1, so we omit it here.

\section{Proofs of Theorems 1.1 and 1.2}

In this section, we obtain the well-posedness and large time behavior of solutions to (1.1) for $l q \in[m, m+1]$, and we prove Theorems 1.1-1.2. To do this, we first obtain the wellposedness to (1.1) by the following proposition.

Proposition 3.1 Assume that $l \geq m>1,1 \leq q<2, l q \in[m, m+1]$, and $u_{0}(x) \in C_{0}(\bar{\Omega})$ satisfies (1.2). Then there exists a unique solution $u(x, t)$ to $(1.1)$ in the sense of Definition 1.1.

Proof The idea of the proof is same as in $[25,28]$, so we omit here.

Next, in order to prove the large time behavior of the solution to (1.1), we shall need several steps: Step 1, we will find that the temporal decay rate of $\|u(t)\|_{\infty}$ is indeed $t^{-\frac{1}{m-1}}$. Step 2, we prove the boundary estimates for the large time which guarantee that no loss of boundary condition occurs throughout the time evolution. Step 3, the half-relaxed limits technique is applied to show the expected convergence after introducing self-similar variables. The approach is developed by Laurençot and Stinner in $[25,27]$. To do this, we need the following lemmas.

Lemma 3.1 (Upper bound) Assume that $l \geq m>1,1 \leq q<2, l q=m$, and the initial data $u_{0}(x) \in W^{1, \infty}(\Omega)$ satisfies (1.2). Then there exists $C_{1}>0$ depending only on $m, l, q, \Omega$, and $\left\|u_{0}\right\|_{\infty}$ such that

$$
u(t, x) \leq C_{1}(1+t)^{-\frac{1}{m-1}}, \quad(t, x) \in(0, \infty) \times \bar{\Omega} .
$$

Proof Assume that $x_{0} \notin \bar{\Omega}$ and $R_{0}>0$ such that $\Omega \subset B\left(x_{0}, R_{0}\right)$. For $(t, x) \in(0, \infty) \times \bar{\Omega}$, we define the function

$$
S_{1}(t, x)=A_{1}(1+t)^{-\frac{1}{m-1}} \varphi(r), \quad \varphi(r)=\left[\frac{m}{m+1}\left(e^{\frac{R(m+1)}{m}}-e^{\frac{r(m+1)}{m}}\right)\right]^{\frac{1}{m}} \quad \text { with } r=\left|x-x_{0}\right|,
$$

where $R>R_{0}$ and $A_{1}>0$ satisfies the following condition:

$$
A_{1} \geq \max \left\{\left(\frac{m}{m-1} e^{\frac{R(m+1)}{m^{2}}}\right)^{\frac{1}{m-1}}, \frac{\left\|u_{0}\right\|_{\infty}}{\varphi\left(R_{0}\right)}\right\} .
$$


Since $x_{0} \notin \bar{\Omega}$, the function $S_{1}(t, x)$ is $C^{\infty}$-smooth in $[0, \infty) \times \bar{\Omega}$. Moreover, according the condition $l \geq m>1,1 \leq q<2$, and $l q=m$, we have $l=m>1$ and $q=1$. Therefore, for $(t, x) \in(0, \infty) \times \Omega$ and $r=\left|x-x_{0}\right|<R_{0}<R$, it follows from (3.2) that

$$
\begin{aligned}
(1 & +t)^{\frac{m}{m-1}}\left\{\partial_{t} S_{1}-\triangle S_{1}^{m}-\left|\nabla S_{1}^{l}\right|^{q}\right\}(t, x) \\
& =-\frac{A_{1}}{m-1} \varphi(r)-A_{1}^{m}\left[\frac{N-1}{r}\left(\varphi^{m}\right)_{r}+\left(\varphi^{m}\right)_{r r}\right]-A_{1}^{m}\left|\left(\varphi^{l}\right)_{r}\right|^{q} \\
& =-\frac{A_{1}}{m-1} \varphi(r)+A_{1}^{m}\left[\frac{N-1}{r} e^{\frac{r(m+1)}{m}}+\frac{m+1}{m} e^{\frac{r(m+1)}{m}}\right]-A_{1}^{m} e^{\frac{r(m+1)}{m}} \\
& \geq A_{1}\left[A_{1}^{m-1}\left(\frac{N-1}{r}+\frac{1}{m}\right) e^{\frac{r(m+1)}{m}}-\frac{1}{m-1} e^{\frac{R(m+1)}{m^{2}}}\right] \\
& \geq A_{1}\left(\frac{1}{m} A_{1}^{m-1}-\frac{1}{m-1} e^{\frac{R(m+1)}{m^{2}}}\right) \\
& \geq 0 .
\end{aligned}
$$

Hence, the condition (3.2) guarantees that $S_{1}(t, x)$ is a supersolution to $(1.1)$ in $(0, \infty) \times \Omega$. In addition, since $r=\left|x-x_{0}\right|<R_{0}<R$ for $x \in \Omega$, for $(t, x) \in(0, \infty) \times \partial \Omega$, we deduce from (3.2) that

$$
u(t, x)=0 \leq A_{1}(1+t)^{-\frac{1}{m-1}} \varphi\left(R_{0}\right) \leq S_{1}(t, x)
$$

and

$$
u_{0}(x) \leq\left\|u_{0}(x)\right\|_{\infty} \leq A_{1} \varphi\left(R_{0}\right) \leq S_{1}(0, x), \quad x \in \bar{\Omega}
$$

By the comparison principle, we have

$$
u(t, x) \leq S_{1}(t, x), \quad(t, x) \in[0, \infty) \times \bar{\Omega} .
$$

The proof of Lemma 3.1 is complete.

Lemma 3.2 (Upper bound) Assume that $l \geq m>1,1 \leq q<2, l q \in(m, m+1]$, and the initial data $u_{0}(x) \in W^{1, \infty}(\Omega)$ satisfies (1.2). Then there exists $C_{2}>0$ depending only on $m$, $l, q, \Omega$, and $\left\|u_{0}\right\|_{\infty}$ such that

$$
u(t, x) \leq C_{2}(1+t)^{-\frac{1}{m-1}}, \quad(t, x) \in(0, \infty) \times \bar{\Omega} .
$$

Proof Assume that $x_{0} \notin \bar{\Omega}$ and $R_{0}>0$ such that $\Omega \subset B\left(x_{0}, R_{0}\right)$. For $(t, x) \in(0, \infty) \times \bar{\Omega}$, we define the function

$$
S_{2}(t, x)=A_{2}(1+\delta t)^{-\frac{1}{m-1}} \sigma(r), \quad \sigma(r)=\left[\frac{m}{m+1}\left(R^{\frac{m+1}{m}}-r^{\frac{m+1}{m}}\right)\right]^{\frac{1}{m}} \quad \text { with } r=\left|x-x_{0}\right|
$$


where the positive constants $A_{2}, R$, and $\delta$ satisfy the following condition:

$$
\begin{aligned}
& A_{2}=\left[\frac{m^{q-1}(1+m(N-1))}{\left.2 l^{q} R^{\frac{(m+1)(l-m) q+m(q+m-1)}{m^{2}}}\right]^{\frac{1}{q-m}}, \quad R=\left[R_{0}^{\frac{m+1}{m}}+\frac{(m+1)\left\|u_{0}\right\|_{\infty}^{m}}{m A_{2}^{m}}\right]^{\frac{m}{m+1}}>R_{0}}\right. \\
& \text { and } \delta=\frac{(m-1)(1+m(N-1)) A_{2}^{m-1}}{2 m R^{\frac{1+m^{2}}{m^{2}}}} .
\end{aligned}
$$

Since $x_{0} \notin \bar{\Omega}$, the function $S_{2}(t, x)$ is $C^{\infty}$-smooth in $[0, \infty) \times \bar{\Omega}$. Moreover, for $(t, x) \in$ $(0, \infty) \times \Omega$ and $r=\left|x-x_{0}\right|<R_{0}<R$, it follows from (3.5) and $l q>m$ that

$$
\begin{aligned}
(1+ & \delta t)^{\frac{m}{m-1}}\left\{\partial_{t} S_{2}-\Delta S_{2}^{m}-\left|\nabla S_{2}^{l}\right|^{q}\right\}(t, x) \\
= & -\frac{A_{2} \delta}{m-1} \sigma(r)-A_{2}^{m}\left[\frac{N-1}{r}\left(\sigma^{m}\right)_{r}+\left(\sigma^{m}\right)_{r r}\right]-A_{2}^{l q}(1+\delta t)^{-\frac{l q-m}{m-1}}\left|\left(\sigma^{l}\right)_{r}\right|^{q} \\
= & -\frac{A_{2} \delta}{m-1} \sigma(r)+A_{2}^{m}\left[\frac{1}{m}+N-1\right] r^{-\frac{m-1}{m}}-A_{2}^{l q}(1+\delta t)^{-\frac{l q-m}{m-1}}\left|\left(\left(\sigma^{m}\right)^{\frac{l}{m}}\right)_{r}\right|^{q} \\
= & -\frac{A_{2} \delta}{m-1} \sigma(r)+A_{2}^{m}\left[\frac{1}{m}+N-1\right] r^{-\frac{m-1}{m}}-A_{2}^{l q}\left(\frac{l}{m}\right)^{q} R^{\frac{(m+1)(l-m) q+m q}{m^{2}}} \\
\geq & A_{2}^{m}\left[\left(N-1+\frac{1}{m}\right) R^{-\frac{m-1}{m}}-A_{2}^{l q-m}\left(\frac{l}{m}\right)^{q} R^{\frac{(m+1)(l-m) q+m q}{m^{2}}}-\frac{\delta R^{\frac{m+1}{m^{2}}}}{(m-1) A_{2}^{m-1}}\right] \\
\geq & A_{2}^{m}\left[\left(\frac{1}{2}\left(N-1+\frac{1}{m}\right) R^{-\frac{m-1}{m}}-A_{2}^{l q-m}\left(\frac{l}{m}\right)^{q} R^{\frac{(m+1)(l-m) q+m q}{m^{2}}}\right)\right. \\
& \left.+\left(\frac{1}{2}\left(N-1+\frac{1}{m}\right) R^{-\frac{m-1}{m}}-\frac{\delta R^{\frac{m+1}{m^{2}}}}{(m-1) A_{2}^{m-1}}\right)\right] \\
\geq & 0 .
\end{aligned}
$$

Therefore, the condition (3.5) guarantees that $S_{2}(t, x)$ is a supersolution to (1.1) in $(0, \infty) \times \Omega$. In addition, since $r=\left|x-x_{0}\right|<R_{0}<R$ for $x \in \Omega$, for $(t, x) \in(0, \infty) \times \partial \Omega$, we deduce from (3.5) that

$$
u(t, x)=0 \leq A_{2}(1+t)^{-\frac{1}{m-1}} \sigma\left(R_{0}\right) \leq S_{2}(t, x),
$$

and

$$
u_{0}(x) \leq\left\|u_{0}(x)\right\|_{\infty} \leq A_{2} \sigma\left(R_{0}\right) \leq S_{2}(0, x), \quad x \in \bar{\Omega} .
$$

By the comparison principle, we have

$$
u(t, x) \leq S_{2}(t, x), \quad(t, x) \in[0, \infty) \times \bar{\Omega} .
$$

The proof of Lemma 3.2 is complete.

Lemma 3.3 (Hölder estimate) Assume that $l \geq m>1,1 \leq q<2, l q \in[m, m+1]$, and the initial data $u_{0}(x) \in W^{1, \infty}(\Omega)$ satisfies (1.2). Then there exists $L_{1}>0$ depending only on $m$, 
$l, q, \Omega$, and $\left\|u_{0}\right\|_{W^{1, \infty}(\Omega)}$ such that

$$
\begin{gathered}
|u(t, x)|=\left|u(t, x)-u\left(t, x_{0}\right)\right| \leq \frac{L_{1}}{(1+t)^{\frac{1}{m-1}}}\left|x-x_{0}\right|^{\frac{1}{m}}, \\
\left(t, x, x_{0}\right) \in[0, \infty) \times \bar{\Omega} \times \partial \Omega .
\end{gathered}
$$

Proof Since the boundary $\partial \Omega$ of $\Omega$ is smooth, there exists $R_{\Omega}>0$ such that for each $x_{0} \in$ $\partial \Omega$, there exists $y_{0} \in \mathbb{R}^{N}$ satisfying $\left|x_{0}-y_{0}\right|=R_{\Omega}$ and $B\left(y_{0}, R_{\Omega}\right) \cap \Omega=\emptyset$. It follows from the initial data condition $u_{0}(x) \in W^{1, \infty}(\Omega)$ that $u_{0}(x)$ is Lipschitz continuous, i.e., there exists $L_{0}>0$ such that

$$
\left|u_{0}(x)-u_{0}(y)\right| \leq L_{0}|x-y|, \quad(x, y) \in \bar{\Omega} \times \bar{\Omega} .
$$

Next, we define the open subset $U_{\kappa, x_{0}}$ of $\mathbb{R}^{N}$ by

$$
U_{\kappa, x_{0}}=\left\{x \in \Omega: R_{\Omega}<\left|x-y_{0}\right|<R_{\Omega}+\kappa\right\}
$$

where $0<\kappa<1$ satisfies $\Omega_{\kappa}=\{x \in \Omega: d(x, \partial \Omega)>\kappa\} \neq \emptyset$, and denote the function

$$
S_{\kappa, x_{0}}(t, x)=A_{3}(1+t)^{-\frac{1}{m-1}} \psi(r), \quad \psi(r)=\left(1-e^{-\frac{r}{\kappa}}\right)^{\frac{1}{m}} \quad \text { with } r=\left|x-y_{0}\right|-R_{\Omega},
$$

for $(t, x) \in(0, \infty) \times \bar{U}_{\kappa, x_{0}}$ and $A_{3}>0$.

Moreover, we assume that

$$
A_{3} \geq \max \left\{e L_{0}, \frac{e^{\frac{1}{m}} C}{(e-1)^{\frac{1}{m}}},\left(\frac{3 e}{m-1}\right)^{\frac{1}{m-1}}\right\}
$$

and

$$
0<\kappa<\min \left\{1, \frac{R_{\Omega}}{3(N-1)},\left(\frac{m^{q}}{3 l^{q} A^{l q-m}}\right)^{\frac{1}{2-q}}\right\}
$$

where $C=\max \left\{C_{1}, C_{2}\right\}>0, C_{1}$, and $C_{2}$ are defined in Lemmas 3.1 and 3.2, respectively.

Since $y_{0} \notin \bar{U}_{\kappa, x_{0}}$, the function $S_{\kappa, x_{0}}(t, x)$ is $C^{\infty}$-smooth in $[0, \infty) \times \bar{U}_{\kappa, x_{0}}$. For $(t, x) \in$ $(0, \infty) \times U_{\kappa, x_{0}}$, we have $r=\left|x-y_{0}\right|-R_{\Omega} \in(0, \kappa)$. By a direct computation, we infer from (3.9)-(3.10) that

$$
\begin{aligned}
& \frac{(1+t)^{\frac{m}{m-1}}}{A_{3}^{m}}\left\{\partial_{t} S_{\kappa, x_{0}}-\Delta S_{\kappa, x_{0}}^{m}-\left|\nabla S_{\kappa, x_{0}}^{l}\right|^{q}\right\}(t, x) \\
&=-\frac{1}{(m-1) A_{3}^{m-1}} \psi(r)-\left[\frac{N-1}{r+R_{\Omega}}\left(\psi^{m}\right)_{r}+\left(\psi^{m}\right)_{r r}\right]-A_{3}^{l q-m}(1+t)^{-\frac{l q-m}{m-1}}\left|\left(\psi^{l}\right)_{r}\right|^{q} \\
&=-\frac{1}{(m-1) A_{3}^{m-1}}\left(1-e^{-\frac{r}{\kappa}}\right)^{\frac{1}{m}}+\frac{1}{\kappa^{2}} e^{-\frac{r}{\kappa}}-\frac{N-1}{\left(r+R_{\Omega}\right) \kappa} e^{-\frac{r}{\kappa}} \\
& \quad-A_{3}^{l q-m}(1+t)^{-\frac{l q-m}{m-1}}\left|\left(\left(\psi^{m}\right)^{\frac{l}{m}}\right)_{r}\right|^{q} \\
& \geq-\frac{1}{(m-1) A_{3}^{m-1}}+\frac{1}{\kappa^{2}} e^{-\frac{r}{\kappa}}-\frac{N-1}{\kappa R_{\Omega}} e^{-\frac{r}{\kappa}}-A_{3}^{l q-m}\left|\left(\left(\psi^{m}\right)^{\frac{l}{m}}\right)_{r}\right|^{q}
\end{aligned}
$$




$$
\begin{aligned}
& \geq \frac{1}{\kappa^{2}} e^{-\frac{r}{\kappa}}\left[1-\frac{N-1}{R_{\Omega}} \kappa-A_{3}^{l q-m}\left(\frac{l}{m}\right)^{q} \kappa^{2-q}-\frac{e}{(m-1) A_{3}^{m-1}}\right] \\
& \geq \frac{1}{\kappa^{2}} e^{-\frac{r}{\kappa}}\left[\left(\frac{1}{3}-\frac{N-1}{R_{\Omega}} \kappa\right)+\left(\frac{1}{3}-A_{3}^{l q-m}\left(\frac{l}{m}\right)^{q} \kappa^{2-q}\right)+\left(\frac{1}{3}-\frac{e}{(m-1) A_{3}^{m-1}}\right)\right]
\end{aligned}
$$

$\geq 0$.

Therefore, $S_{\kappa, x_{0}}(t, x)$ is a supersolution to $(1.1)$ in $(0, \infty) \times U_{\kappa, x_{0}}$. In addition, it follows from (3.8)-(3.10) and the mean value theorem that

$$
\begin{aligned}
S_{\kappa, x_{0}}(0, x) & =A_{3}\left(1-e^{-\frac{\left|x-y_{0}\right|-R_{\Omega}}{\kappa}}\right)^{\frac{1}{m}} \geq A_{3}\left(1-e^{-\frac{\left|x-y_{0}\right|-R_{\Omega}}{\kappa}}\right) \\
& \geq A_{3} \frac{\left|x-y_{0}\right|-R_{\Omega}}{e \kappa}=\frac{A_{3}}{e \kappa} \operatorname{dist}\left(x, \partial B\left(y_{0}, R_{\Omega}\right)\right) \\
& \geq \frac{A_{3}}{e \kappa} \operatorname{dist}(x, \partial \Omega) \geq L_{0} \operatorname{dist}(x, \partial \Omega) \geq u_{0}(x), \quad x \in \bar{U}_{\delta, x_{0}} .
\end{aligned}
$$

Moreover, for $(t, x) \in(0, \infty) \times \partial U_{\kappa, x_{0}}$, we have either $x \in \partial \Omega$ or $r=\left|x-y_{0}\right|-R_{\Omega}=\delta$. If $x \in \partial \Omega$, then we have

$$
S_{\kappa, x_{0}}(t, x)=A_{3}(1+t)^{-\frac{1}{m-1}}\left(1-e^{-\frac{r}{\kappa}}\right)^{\frac{1}{m}} \geq 0=u(x, t), \quad(t, x) \in(0, \infty) \times \partial \Omega .
$$

If $r=\left|x-y_{0}\right|-R_{\Omega}=\delta$, by Lemmas 3.1-3.2 and (3.9), we have

$$
S_{\kappa, x_{0}}(t, x)=A_{3}(1+t)^{-\frac{1}{m-1}}\left(1-e^{-1}\right)^{\frac{1}{m}} \geq C(1+t)^{-\frac{1}{m-1}} \geq u(t, x) .
$$

By the comparison principle [6] we have

$$
u(t, x) \leq S_{\kappa, x_{0}}(t, x), \quad(t, x) \in[0, \infty) \times \bar{U}_{\kappa, x_{0}} .
$$

Consequently,

$$
0 \leq u(t, x)-u\left(t, x_{0}\right)=u(t, x) \leq A_{3}(1+t)^{-\frac{1}{m-1}}\left(1-e^{-\frac{\left|x-y_{0}\right|-R_{\Omega}}{\kappa}}\right)^{\frac{1}{m}}
$$

for $(t, x) \in[0, \infty) \times \bar{U}_{\kappa, x_{0}}$.

Since $\left|x_{0}-y_{0}\right|-R_{\Omega}=0$, we have

$$
0 \leq\left|u(t, x)-u\left(t, x_{0}\right)\right| \leq \frac{A_{3}}{\kappa^{\frac{1}{m}}(1+t)^{\frac{1}{m-1}}}\left|x-x_{0}\right|^{\frac{1}{m}}, \quad x \in \bar{U}_{\kappa, x_{0}} .
$$

Finally, we consider $x \in \Omega$ and $x_{0} \in \partial \Omega$. If $\left|x-x_{0}\right| \geq \frac{\kappa}{2}$, it follows from Lemmas 3.1-3.2 that

$$
\left|u(t, x)-u\left(t, x_{0}\right)\right|=u(t, x) \leq \frac{2^{\frac{1}{m}} C}{\kappa^{\frac{1}{m}}(1+t)^{\frac{1}{m-1}}}\left|x-x_{0}\right|^{\frac{1}{m}}
$$

where $C=\max \left\{C_{1}, C_{2}\right\}>0$, and $C_{1}$ and $C_{2}$ are defined in Lemmas 3.1 and 3.2, respectively.

If $\left|x-x_{0}\right|<\frac{\kappa}{2}$, let $y_{0} \in \mathbb{R}^{N}$ satisfy $\left|x_{0}-y_{0}\right|=R_{\Omega}$ and $B\left(y_{0}, R_{\Omega}\right) \cap \Omega=\emptyset$. Since $x \in \Omega$, we have

$$
\left|x-y_{0}\right|>R_{\Omega} \text { and }\left|x-y_{0}\right| \leq\left|x-x_{0}\right|+\left|x_{0}-y_{0}\right|<R_{\Omega}+\kappa,
$$


which implies $x \in U_{\kappa, x_{0}}$. Therefore, we deduce from (3.11) that

$$
\left|u(t, x)-u\left(t, x_{0}\right)\right|=u(t, x) \leq \frac{A_{3}}{\kappa^{\frac{1}{m}}(1+t)^{\frac{1}{m-1}}}\left|x-x_{0}\right|^{\frac{1}{m}} .
$$

Choosing $L_{1}=\max \left\{\frac{2^{\frac{1}{m}} C}{\kappa^{\frac{1}{m}}}, \frac{A_{3}}{\kappa^{\frac{1}{m}}}\right\}$, we have

$$
|u(t, x)|=\left|u(t, x)-u\left(t, x_{0}\right)\right| \leq \frac{L_{1}}{(1+t)^{\frac{1}{m-1}}}\left|x-x_{0}\right|^{\frac{1}{m}}, \quad\left(t, x, x_{0}\right) \in[0, \infty) \times \bar{\Omega} \times \partial \Omega .
$$

The proof of Lemma 3.3 is complete.

We next proceed as in [29] to deduce the Hölder continuity of $u(x, t)$ from Lemma 3.3. Therefore, we obtain the following corollary.

Corollary 3.1 Assume that $l \geq m>1,1 \leq q<2, l q \in[m, m+1]$, and the initial data $u_{0}(x) \in W^{1, \infty}(\Omega)$ satisfies (1.2). Then there exists $L_{2}>0$ depending only on $m, l, q, \Omega$, and $\left\|u_{0}\right\|_{W^{1, \infty}(\Omega)}$ such that

$$
|u(t, x)-u(t, y)| \leq \frac{L_{2}}{(1+t)^{\frac{1}{m-1}}}|x-y|^{\frac{1}{m}}, \quad(t, x, y) \in[0, \infty) \times \bar{\Omega} \times \bar{\Omega} .
$$

Proof The proof is similar to the argument in [25, 29], so we omit here.

Proofs of Theorems 1.1 and 1.2 The proofs are based on the ideas in [25], but we give the details of the argument for the reader's convenience. Let $U(x, t)$ be the solution to the porous medium equation with homogeneous Dirichlet boundary conditions

$$
\begin{cases}\partial_{t} U-\Delta U^{m}=0, & (t, x) \in(0, \infty) \times \Omega \\ U(t, x)=0, & (t, x) \in(0, \infty) \times \partial \Omega \\ U(0, x)=u_{0}(x), & x \in \Omega\end{cases}
$$

According to the nonnegativity of $\left|\nabla u^{l}\right|^{q}$, it follows from the comparison principle [6] that

$$
0 \leq U(t, x) \leq u(t, x), \quad(t, x) \in(0, \infty) \times \bar{\Omega} .
$$

We introduce the scaling variable $s=\ln t$ for $t>0$ and denote the new unknown function $v$ and $V$ by

$$
u(t, x)=t^{-\frac{1}{m-1}} v(\ln t, x), \quad(t, x) \in(0, \infty) \times \bar{\Omega}
$$

and

$$
U(t, x)=t^{-\frac{1}{m-1}} V(\ln t, x), \quad(t, x) \in(0, \infty) \times \bar{\Omega} .
$$


Then $v$ is a viscosity solution to the following problem:

$$
\begin{cases}\partial_{s} v-\Delta v^{m}-e^{-\frac{(l q-m) s}{m-1}}\left|\nabla v^{l}\right|^{q}-\frac{v}{m-1}=0, & (s, x) \in(0, \infty) \times \Omega, \\ v(s, x)=0, & (s, x) \in(0, \infty) \times \partial \Omega, \\ v(0, x)=u(1, x), & x \in \Omega .\end{cases}
$$

In addition, owing to Lemmas 3.1-3.3 and (3.14), we have

$$
V(s, x) \leq v(s, x) \leq C, \quad(s, x) \in[0, \infty) \times \bar{\Omega},
$$

and

$$
|v(s, x)-v(s, y)| \leq L_{1}|x-y|^{\frac{1}{m}}, \quad(s, x, y) \in[0, \infty) \times \bar{\Omega} \times \partial \Omega .
$$

Next, for $\varepsilon \in(0,1)$, we define

$$
w_{\varepsilon}(s, x)=v\left(\frac{s}{\varepsilon}, x\right), \quad(s, x) \in[0, \infty) \times \bar{\Omega},
$$

and the half-relaxed limits

$$
w_{*}(x)=\lim _{(\sigma, y, \varepsilon) \rightarrow(s, x, 0)} \inf w_{\varepsilon}(\sigma, y) \quad \text { and } \quad w^{*}(x)=\lim _{(\sigma, y, \varepsilon) \rightarrow(s, x, 0)} \sup w_{\varepsilon}(\sigma, y) \text {, }
$$

for $(s, x) \in(0, \infty) \times \bar{\Omega}$.

By (3.16), it is easy to see that $w_{*}(x)$ and $w^{*}(x)$ are well-defined and do not depend on $s>0$. Moreover, it readily follows from (3.15) and (3.17) that

$$
w_{*}(x)=w^{*}(x)=0, \quad x \in \partial \Omega .
$$

By a direct computation, $w_{\varepsilon}(s, x)$ is a solution to the following initial-boundary problem:

$$
\begin{cases}\varepsilon \partial_{s} w_{\varepsilon}-\Delta w_{\varepsilon}^{m}-e^{-\frac{(l q-m) s}{(m-1) \varepsilon}}\left|\nabla w_{\varepsilon}^{l}\right|^{q}-\frac{w_{\varepsilon}}{m-1}=0, & (s, x) \in(0, \infty) \times \Omega, \\ w_{\varepsilon}(s, x)=0, & (s, x) \in(0, \infty) \times \partial \Omega, \\ w_{\varepsilon}(0, x)=u(1, x), & x \in \Omega .\end{cases}
$$

Next, we shall give proofs of Theorems 1.1 and 1.2. To do this, we distinguish the two cases $l q=m$ and $l q \in(m, m+1]$.

Case $1 . l q=m$. We use the stability of semicontinuous viscosity solutions [6] to deduce from (3.19) that

$$
w_{*}(x) \text { is a supersolution to }(2.1) \text { in } \Omega
$$

and 
In addition, by [30], $V(s) \rightarrow f_{0}$ in $L^{\infty}(\Omega)$ as $s \rightarrow \infty$. Moreover, it follows from (3.16) and the definition of $w_{*}(x)$ and $w^{*}(x)$ that

$$
f_{0}(x) \leq w_{*}(x) \leq w^{*}(x) \leq C, \quad x \in \bar{\Omega} .
$$

Since $f_{0}>0$ in $\Omega$ by [30], we deduce from (3.22) that $w_{*}(x)$ and $w^{*}(x)$ are positive and bounded in $\Omega$ and vanish on $\partial \Omega$ by (3.18). Owing to (3.20) and (3.21), we infer from Lemma 2.1 that

$$
w^{*}(x) \leq w_{*}(x), \quad x \in \bar{\Omega} .
$$

By (3.22), we have

$$
w^{*}(x)=w_{*}(x), \quad x \in \bar{\Omega} .
$$

Setting $f(x)=w_{*}(x)=w^{*}(x)$, we deduce from (3.18), (3.20), (3.21), and (3.22) that $f(x) \in$ $C_{0}(\bar{\Omega})$ is a positive viscosity solution to (2.1) and solves (1.9). Therefore, the existence of a positive solution to (1.9) is proved. Moreover, by Corollary 2.1, we obtain the uniqueness of solution to (1.9).

Furthermore, it follows from the equality $w_{*}(x)=w^{*}(x)$ that

$$
\left\|w_{\varepsilon}(1)-f\right\|_{\infty} \rightarrow 0 \quad \text { as } \varepsilon \rightarrow 0
$$

i.e.,

$$
\lim _{s \rightarrow \infty}\|v(s)-f\|_{\infty}=0
$$

Therefore, we infer from the scaling transformation that

$$
\lim _{t \rightarrow \infty}\left\|t^{\frac{1}{m-1}} u(t)-f\right\|_{\infty}=0
$$

Finally, Corollary 3.1 gives the last statement of Theorem 1.1. The proof of Theorem 1.1 is complete.

Case 2. $l q \in(m, m+1]$. We use once more the stability of semicontinuous viscosity solutions [6] to deduce from (3.19) that

$$
w_{*}(x) \text { is a supersolution to }(2.10) \text { in } \Omega
$$

and

$w^{*}(x)$ is a subsolution to $(2.10)$ in $\Omega$.

In addition, by [30], $V(s) \rightarrow f_{0}$ in $L^{\infty}(\Omega)$ as $s \rightarrow \infty$. Moreover, it follows from (3.16) and the definition of $w_{*}(x)$ and $w^{*}(x)$ that

$$
f_{0}(x) \leq w_{*}(x) \leq w^{*}(x) \leq C, \quad x \in \bar{\Omega} .
$$


Since $f_{0}>0$ in $\Omega$ by [30] and is a solution to (2.10), we can apply Lemma 2.2 to conclude that $w_{*}(x) \leq f_{0}$ in $\bar{\Omega}$. Owing to (3.27), we have

$$
w_{*}(x)=w^{*}(x)=f_{0}(x), \quad x \in \bar{\Omega} .
$$

Therefore, we deduce from (3.19) that

$$
\left\|w_{\varepsilon}(1)-f_{0}\right\|_{\infty} \rightarrow 0 \quad \text { as } \varepsilon \rightarrow 0,
$$

i.e.,

$$
\lim _{s \rightarrow \infty}\left\|v(s)-f_{0}\right\|_{\infty}=0
$$

Thus, we infer from the scaling transformation that

$$
\lim _{t \rightarrow \infty}\left\|t^{\frac{1}{m-1}} u(t)-f_{0}\right\|_{\infty}=0
$$

The proof of Theorem 1.2 is complete.

\section{Proof of Theorem 1.3}

In this section, we shall consider the well-posedness and the large time behavior of solutions to (1.1) with the small initial data $u_{0}(x)$ for $l q>m+1$ by the method used in [25]. To do this, we need the following lemma.

Lemma 4.1 Let $l \geq m>1$ and $1 \leq q<2$. Assume that $G$ is the corresponding solution to (1.1) with the initial data $G_{0} \in W^{1, \infty}(\Omega)$ satisfying (1.2) for the case $l q=m$, and denote

$$
\mathcal{F}(t, x)=\frac{1}{\ell\left[G_{0}\right]} G\left(\frac{t}{\ell\left[G_{0}\right]^{m-1}}, x\right), \quad(t, x) \in[0, \infty) \times \bar{\Omega},
$$

where the parameter $\ell\left[G_{0}\right]$ is defined in (1.10). Then $\mathcal{F}(t, x)$ is a solution to (1.1) with the initial data $G_{0} / \ell\left[G_{0}\right]$ and $l q=m$ such that $\left|\nabla \mathcal{F}^{l}\right| \leq 1$ for $(t, x) \in[0, \infty) \times \bar{\Omega}$. Moreover, $\mathcal{F}(t, x)$ is a supersolution to (1.1) for $l q>m$.

Proof According to the definition of $\ell\left[G_{0}\right]$ in (1.10), it is easy to see that $\left|\nabla \mathcal{F}^{l}\right| \leq 1$ for $(t, x) \in[0, \infty) \times \bar{\Omega}$.

Next, let $\varphi(t, x) \in C^{2}((0, \infty) \times \Omega)$ and $\mathcal{F}-\varphi$ has a local minimum at $\left(t_{0}, x_{0}\right) \in(0, \infty) \times \Omega$. Since $\mathcal{F}$ is smooth with respect to the time variable and Hölder continuous with respect to the space variable, we obtain

$$
\left|\nabla \varphi^{l}\left(t_{0}, x_{0}\right)\right| \leq 1
$$

Moreover, introducing $\psi(t, x):=\ell\left[G_{0}\right] \varphi\left(\ell\left[G_{0}\right]^{m-1} t, x\right)$ for $(t, x) \in[0, \infty) \times \bar{\Omega}$, the function $G-\psi$ has a local minimum at $\left(t_{0} \ell\left[G_{0}\right]^{1-m}, x_{0}\right)$ such that

$$
\partial_{t} \psi\left(t_{0} \ell\left[G_{0}\right]^{1-m}, x_{0}\right)-\Delta \psi^{m}\left(t_{0} \ell\left[G_{0}\right]^{1-m}, x_{0}\right)-\left|\nabla \psi^{l}\left(t_{0} \ell\left[G_{0}\right]^{1-m}, x_{0}\right)\right|^{\frac{m}{l}} \geq 0,
$$


i.e.,

$$
\partial_{t} \varphi\left(t_{0}, x_{0}\right)-\Delta \varphi^{m}\left(t_{0}, x_{0}\right)-\left|\nabla \varphi^{l}\left(t_{0}, x_{0}\right)\right|^{\frac{m}{T}} \geq 0 .
$$

Therefore, $\mathcal{F}(t, x)$ is a supersolution to (1.1) with $l q=m$. In a similar way, it can be shown that $\mathcal{F}(t, x)$ is also a subsolution. Hence, $\mathcal{F}(t, x)$ is a solution to (1.1) with $l q=m$.

Furthermore, we deduce from (4.2), (4.3), and $l q>m$ that

$$
\begin{aligned}
& \partial_{t} \varphi\left(t_{0}, x_{0}\right)-\Delta \varphi^{m}\left(t_{0}, x_{0}\right)-\left|\nabla \varphi^{l}\left(t_{0}, x_{0}\right)\right|^{q} \\
& \quad \geq\left|\nabla \varphi^{l}\left(t_{0}, x_{0}\right)\right|^{\frac{m}{T}}\left(1-\left|\nabla \varphi^{l}\left(t_{0}, x_{0}\right)\right|^{q-\frac{m}{T}}\right) \geq 0 .
\end{aligned}
$$

The proof of Lemma 4.1 is complete.

Proposition 4.1 Let $l \geq m>1,1 \leq q<2$, and $l q>m+1$. Assume that the initial data $u_{0}(x) \in W^{1, \infty}(\Omega)$ satisfies (1.2), moreover, there exists $G_{0} \in W^{1, \infty}(\Omega)$ satisfying (1.2) such that

$$
u_{0}(x) \leq \frac{G_{0}(x)}{\ell\left[G_{0}\right]}, \quad x \in \bar{\Omega}
$$

where the parameter $\ell\left[G_{0}\right]$ is defined in (1.10). Then there exists a unique solution $u$ to (1.1) in the sense of Definition 1.1 and it satisfies

$$
u(t, x) \leq \mathcal{F}(t, x), \quad(t, x) \in[0, \infty) \times \bar{\Omega},
$$

where $\mathcal{F}(t, x)$ is defined in (4.1).

Proof On the one hand, the solution $U$ to the porous medium equation (3.13) is clearly a subsolution to $(1.1)$ in $(0, \infty) \times \Omega$.

On the other hand, it follows from Lemma 4.1 that the function $\mathcal{F}(t, x)$ is a supersolution to (1.1) in $(0, \infty) \times \Omega$. Therefore, $\mathcal{F}(t, x)$ is a supersolution to (3.13).

Since $U=\mathcal{F}=0$ on $(0, \infty) \times \partial \Omega$ and $U(0, x)=u_{0}(x) \leq \mathcal{F}(0, x)$ for $x \in \bar{\Omega}$ by (4.5), we infer from the comparison principle [6] that

$$
U(t, x) \leq \mathcal{F}(t, x), \quad(t, x) \in[0, \infty) \times \bar{\Omega} .
$$

This property and the simultaneous vanishing of $U$ and $\mathcal{F}$ on $(0, \infty) \times \partial \Omega$ allow us to use the classical Perron method to establish the existence of a solution $u(x, t)$ to (1.1) in the sense of Definition 1.1 which satisfies (4.6). The uniqueness next follows from the comparison principle [6]. The proof of Proposition 4.1 is complete.

Proof of Theorem 1.3 We notice that Lemma 3.2 is also valid in that case. It readily follows from Lemma 3.3 and Proposition 4.1 that

$$
\begin{aligned}
0 \leq u(t, x) & =u(t, x)-u\left(t, x_{0}\right) \leq \mathcal{F}(t, x) \leq \frac{L_{1}}{(1+t)^{\frac{1}{m-1}}}\left|x-x_{0}\right|^{\frac{1}{m}}, \\
\left(t, x, x_{0}\right) & \in[0, \infty) \times \bar{\Omega} \times \partial \Omega .
\end{aligned}
$$


The convergence proof is similar to that performed in the proof of Theorem 1.2 for $l q \in$ $(m, m+1]$. The proof of Theorem 1.3 is complete.

\section{Proof of Theorem 1.4}

In this section, when $l q>m+1$, we shall prove that the solution $u(x, t)$ of (1.1) blows up in finite time for the large initial data $u_{0}(x)$ in the sense of weak solution by the method used in [26].

In order to obtain a blow-up condition corresponding to (1.1), we have to modify the function $e^{-\varepsilon|x|^{2}}$ used in [31-33], and introduce a test function $\phi_{\varepsilon}(x)$ as follows:

$$
\phi_{\varepsilon}(x)=A_{\varepsilon} e^{-\varepsilon|x|} \quad \text { with } A_{\varepsilon}=\frac{1}{\int_{\Omega} e^{-\varepsilon|x|} d x} .
$$

Proof of Theorem 1.4 Suppose that $u(x, t)$ is the solution of the problem (1.1) and $T$ is the blow-up time of the solution. For $s \in\left(0, \frac{1}{2}\right)$, we denote

$$
W(t)=\frac{1}{s} \int_{\Omega} u^{s} \phi_{\varepsilon}(x) d x, \quad t \in(0, T) .
$$

By a direct calculation, we have

$$
\begin{aligned}
W^{\prime}(t)= & \int_{\Omega} u^{s-1} \phi_{\varepsilon} u_{t} d x \\
= & \int_{\Omega} u^{s-1} \phi_{\varepsilon} \Delta u^{m} d x+\int_{\Omega} u^{s-1} \phi_{\varepsilon}\left|\nabla u^{l}\right|^{q} d x \\
\geq & -(s-1) \int_{\Omega} u^{s-2} \phi_{\varepsilon} \nabla u^{m} \cdot \nabla u d x+\varepsilon \int_{\Omega} u^{s-1} \phi_{\varepsilon} \nabla u^{m} \cdot \frac{x}{|x|} d x \\
& +\int_{\Omega} u^{s-1} \phi_{\varepsilon}\left|\nabla u^{l}\right|^{q} d x \\
\geq & m(1-s) \int_{\Omega} u^{m+s-3} \phi_{\varepsilon}|\nabla u|^{2} d x-m \varepsilon \int_{\Omega} u^{m+s-2} \phi_{\varepsilon}|\nabla u| d x \\
& +\int_{\Omega} u^{s-1} \phi_{\varepsilon}\left|\nabla u^{l}\right|^{q} d x .
\end{aligned}
$$

By Young's inequality, we obtain

$$
\varepsilon \int_{\Omega} u^{m+s-2} \phi_{\varepsilon}|\nabla u| d x \leq \frac{1}{2} \int_{\Omega} u^{m+s-3} \phi_{\varepsilon}|\nabla u|^{2} d x+\frac{\varepsilon^{2}}{2} \int_{\Omega} u^{m+s-1} \phi_{\varepsilon} d x .
$$

Since $0<s<\frac{1}{2}$, it follows from (5.2), (5.3), and Poincarés inequality that

$$
\begin{aligned}
W^{\prime}(t) & \geq \int_{\Omega} u^{s-1} \phi_{\varepsilon}\left|\nabla u^{l}\right|^{q} d x-\frac{m \varepsilon^{2}}{2} \int_{\Omega} u^{m+s-1} \phi_{\varepsilon} d x \\
& =\left(\frac{q l}{q l+s-1}\right)^{q} \int_{\Omega} \phi_{\varepsilon}\left|\nabla u^{\frac{q l+s-1}{q}}\right|^{q} d x-\frac{m \varepsilon^{2}}{2} \int_{\Omega} u^{m+s-1} \phi_{\varepsilon} d x \\
& \geq\left(\frac{q l}{q l+s-1}\right)^{q} \int_{\Omega} \phi_{\varepsilon} u^{q l+s-1} d x-\frac{m \varepsilon^{2}}{2} \int_{\Omega} u^{m+s-1} \phi_{\varepsilon} d x .
\end{aligned}
$$


According to $q l>m+1,0<s<\frac{1}{2}, \int_{\Omega} \phi_{\varepsilon}(x) d x=1$ and Hölder's inequality, we have

$$
\int_{\Omega} u^{m+s-1} \phi_{\varepsilon} d x=\int_{\Omega} u^{m+s-1} \phi_{\varepsilon}^{\frac{m+s-1}{q l+s-1}} \phi_{\varepsilon}^{\frac{q l-m}{q l+s-1}} d x \leq\left(\int_{\Omega} u^{q l+s-1} \phi_{\varepsilon} d x\right)^{\frac{m+s-1}{q l+s-1}} .
$$

Thus, by (5.4) and (5.5), we obtain

$$
\frac{d W}{d t} \geq\left(\int_{\Omega} u^{q l+s-1} \phi_{\varepsilon} d x\right)^{\frac{m+s-1}{q l+s-1}}\left[\left(\frac{q l}{q l+s-1}\right)^{q}\left(\int_{\Omega} u^{q l+s-1} \phi_{\varepsilon} d x\right)^{\frac{q l-m}{q l+s-1}}-\frac{m \varepsilon^{2}}{2}\right]
$$

Owing to $q l>m+1,0<s<\frac{1}{2}, \int_{\Omega} \phi_{\varepsilon}(x) d x=1$, and Jensen's inequality, we have

$$
\left(\int_{\Omega} u^{q l+s-1} \phi_{\varepsilon} d x\right)^{\frac{q l-m}{q l+s-1}} \geq\left(\int_{\Omega} u^{s} \phi_{\varepsilon} d x\right)^{\frac{q l-m}{s}} .
$$

Therefore, it follows from (5.6) and (5.7) that

$$
\frac{d W}{d t} \geq \frac{1}{2}\left(\frac{q l}{q l+s-1}\right)^{q}\left(\int_{\Omega} u^{s} \phi_{\varepsilon} d x\right)^{\frac{q l+s-1}{s}}=\frac{1}{2}\left(\frac{q l}{q l+s-1}\right)^{q} s^{\frac{q l+s-1}{s}} W^{\frac{q l+s-1}{s}}(t)
$$

as long as

$$
W(t) \geq \frac{1}{s}\left(\frac{q l+s-1}{q l}\right)^{\frac{q s}{q l-m}}\left(m \varepsilon^{2}\right)^{\frac{s}{q l-m}} \quad \text { for all } t \in[0, T) .
$$

Taking

$$
K_{0}=\left(\frac{q l+s-1}{q l}\right)^{\frac{q s}{q l-m}}\left(m \varepsilon^{2}\right)^{\frac{s}{q l-m}} .
$$

Since the initial data $u_{0}(x)$ satisfies

$$
\int_{\Omega} u_{0}^{s}(x) \phi_{\varepsilon}(x) d x \geq K_{0}
$$

we have

$$
W(0) \geq \frac{1}{s} K_{0}=\frac{1}{s}\left(\frac{q l+s-1}{q l}\right)^{\frac{q s}{q l-m}}\left(m \varepsilon^{2}\right)^{\frac{s}{q l-m}} .
$$

Therefore, $w(t)$ increases and remains above $\frac{1}{s} K_{0}$ for all $t \in[0, T]$.

By (5.8), integrating over $(0, t)$ yields

$$
W(t) \geq\left(W^{\frac{1-q l}{s}}(0)-K_{1} t\right)^{-\frac{s}{q l-1}} \quad \text { with } K_{1}=\frac{q l-1}{2}\left(\frac{q l}{q l+s-1}\right)^{q} s^{\frac{q l-1}{s}} .
$$

Hence, it follows from (5.10) and (5.11) that the solution $u(x, t)$ of (1.1) blows up in finite time, $T=\frac{1}{K_{1}} W^{\frac{1-q l}{s}}(0)$. 
Moreover, by (5.10), we obtain the upper estimate on the blow-up time $T$ of the solution $u(x, t)$ as follows:

$$
T \leq \frac{2}{q l-1}\left(\frac{q l}{q l+s-1}\right)^{\frac{q(m-1)}{q l-m}}\left(m \varepsilon^{2}\right)^{\frac{1-q l}{q l-m}} .
$$

The proof of Theorem 1.4 is complete.

\section{Competing interests}

The authors declare that they have no competing interests.

\section{Authors' contributions}

The authors declare that the study was realized in collaboration with the same responsibility. All authors read and approved the final manuscript.

\section{Author details}

${ }^{1}$ Department of Applied Mathematics, Southwestern University of Finance and Economics, Chengdu, 610074, P.R. China. ${ }^{2}$ College of Mathematics and Statistics, Chongqing University, Chongqing, 401331, P.R. China. ${ }^{3}$ College of Mathematics and Physics, Chongqing University of Posts and Telecommunications, Chongqing, 400065, P.R. China.

\section{Acknowledgements}

The authors would like to express sincere gratitude to the referees for their valuable suggestions and comments on the original manuscript. The first author is supported by Scientific Research Fund of Sichuan Provincial Science and Technology Department (2014JY0098); the second author is supported in part by the Fundamental Research Funds for the Central Universities, Project No. CDJXS 121000 14; the third author is supported in part by NSF of China (11371384).

\section{Received: 23 December 2013 Accepted: 3 April 2014 Published: 06 May 2014}

\section{References}

1. Andreucci, D: Degenerate parabolic equations with initial data measures. Trans. Am. Math. Soc. 349, 3911-3923 (1997)

2. Gilding, BH, Guedda, M, Kersner, R: The Cauchy problem for $u_{t}=\Delta u+|\nabla u|^{q}$. J. Math. Anal. Appl. 284, $733-755$ (2003)

3. Li, YX, Souplet, P: Single-point gradient blow-up on the boundary for diffusive Hamilton-Jacobi equations in planar domains. Commun. Math. Phys. 293, 499-517 (2010)

4. Bardi, M, Capuzzo-Dolcetta, I: Optimal Control and Viscosity Solutions of Hamilton-Jacobi-Bellman Equations. Birkhäuser, Boston (1997)

5. Barles, G: Solutions de Viscosité des Equations d'Hamilton-Jacobi. Mathématiques et Applications, vol. 17. Springer, Berlin (1994)

6. Crandall, MG, Ishii, H, Lions, PL: User's guide to viscosity solutions of second order partial differential equations. Bull. Am. Math. Soc. (N.S.) 27, 1-67 (1992)

7. Arrieta, JM, Bernal, AR, Souplet, P: Boundedness of global solutions for nonlinear parabolic equations involving gradient blow-up phenomena. Ann. Sc. Norm. Super. Pisa, Cl. Sci. 3, 1-15 (2004)

8. Benachour, SD, Dăbuleanu-Hapca, S, Laurençot, P: Decay estimates for a viscous Hamilton-Jacobi equation with homogeneous Dirichlet boundary conditions. Asymptot. Anal. 51, 209-229 (2007)

9. Ding, JT, Guo, BZ: Global existence and blow-up solutions for quasilinear reaction-diffusion equations with a gradient term. Appl. Math. Lett. 24, 936-942 (2011)

10. Souplet, P, Zhang, QS: Global solutions of inhomogeneous Hamilton-Jacobi equations. J. Anal. Math. 99, 355-396 (2006)

11. Xia, L, Yao, ZA: Existence, uniqueness and asymptotic behavior of solutions for a singular parabolic equation. J. Math. Anal. Appl. 358, 182-188 (2009)

12. Zhou, WS, Lei, PD: A one-dimensional nonlinear heat equation with a singular term. J. Math. Anal. Appl. 368, 711-726 (2010)

13. Barles, G, Souganidis, PE: On the large time behaviour of solutions of Hamilton-Jacobi equations. SIAM J. Math. Anal. 31, 925-939 (2000)

14. Benachour, S, Dabuleanu, S: Large time behavior for a viscous Hamilton-Jacobi equation with Neumann boundary condition. J. Differ. Equ. 216, 223-258 (2005)

15. Laurençot, P: Convergence to steady states for a one-dimensional viscous Hamilton-Jacobi equation with Dirichlet boundary conditions. Pac. J. Math. 230, 347-364 (2007)

16. Namah, G, Roquejoffre, JM: Remarks on the long time behaviour of the solutions of Hamilton-Jacobi equations. Commun. Partial Differ. Equ. 24, 883-893 (1999)

17. Qi, YW, Wang, MX: The self-similar profiles of generalized KPZ equation. Pac. J. Math. 201, 223-240 (2001)

18. Quittner, P, Souplet, P: Superlinear Parabolic Problems. Blow-up, Global Existence and Steady States. Birkhäuser, Basel (2007)

19. Roquejoffre, JM: Convergence to steady states or periodic solutions in a class of Hamilton-Jacobi equations. J. Math. Pures Appl. 80, 85-104 (2001)

20. Shi, PH, Wang, MX: Global solution of the fast diffusion equation with gradient absorption terms. J. Math. Anal. Appl. $326,602-621(2007)$ 
21. Tchamba, TT: Large time behavior of solutions of viscous Hamilton-Jacobi equations with superquadratic Hamiltonian. Asymptot. Anal. 66, 161-186 (2010)

22. Gilding, $\mathrm{BH}$ : The Cauchy problem for $u_{t}=\Delta u+|\nabla u|^{q}$ : large-time behaviour. J. Math. Pures Appl. 84, 753-785 (2005)

23. Stinner, C: Convergence to steady states in a viscous Hamilton-Jacobi equation with degenerate diffusion. J. Differ. Equ. 248, 209-228 (2010)

24. Barles, G, Laurençot, P, Stinner, C: Convergence to steady states for radially symmetric solutions to a quasilinear degenerate diffusive Hamilton-Jacobi equation. Asymptot. Anal. 67, 229-250 (2010)

25. Laurençot, P, Stinner, C: Convergence to separate variable solutions for a degenerate parabolic equation with gradient source. J. Dyn. Differ. Equ. 24, 29-49 (2012)

26. $\mathrm{Mu}, \mathrm{CL}, \mathrm{Li}, \mathrm{YH}, \mathrm{Wang}, \mathrm{Y}$ : Life span and a new critical exponent for a quasilinear degenerate parabolic equation with slow decay initial values. Nonlinear Anal., Real World Appl. 11, 198-206 (2010)

27. Laurençot, P, Stinner, C: Refined asymptotics for the infinite heat equation with homogeneous Dirichlet boundary conditions. Commun. Partial Differ. Equ. 36, 532-546 (2011)

28. Da Lio, F: Comparison results for quasilinear equations in annular domains and applications. Commun. Partial Differ. Equ. 27, 283-323 (2002)

29. Kawohl, B, Kutev, N: Comparison principle and Lipschitz regularity for viscosity solutions of some classes of nonlinear partial differential equations. Funkc. Ekvacioj 43, 241-253 (2000)

30. Manfredi, JJ, Vespri, V: Large time behavior of solutions to a class of doubly nonlinear parabolic equations. Electron. J. Differ. Equ. 2, 1-17 (1994)

31. Guo, JS, Guo, YY: On a fast diffusion equation with source. Tohoku Math. J. 53, 571-579 (2001)

32. Li, YH, Mu, CL: Life span and a new critical exponent for a degenerate parabolic equation. J. Differ. Equ. 207, 392-406 (2004)

33. Mukai, K, Mochizuki, K, Huang, Q: Large time behavior and life span for a quasilinear parabolic equation with slowly decaying initial values. Nonlinear Anal. TMA 39, 33-45 (2000)

10.1186/1687-2770-2014-98

Cite this article as: Li et al.: Large time behavior of solutions for the porous medium equation with a nonlinear gradient source. Boundary Value Problems 2014, 2014:98

\section{Submit your manuscript to a SpringerOpen ${ }^{\odot}$ journal and benefit from:}

- Convenient online submission

- Rigorous peer review

- Immediate publication on acceptance

- Open access: articles freely available online

- High visibility within the field

- Retaining the copyright to your article 